\title{
Double-diffusive mixed convection of pseudoplastic fluids in an inclined square cavity partially heated
}

\author{
Nihal Toudja ${ }^{1,}$, ,Nabila Labsi $^{1}$, Youb Khaled Benkahla ${ }^{1}$, Abdelkader Boutra ${ }^{1}$, Seif-Eddine Ouyahia ${ }^{1}$, and Mahdi \\ Benzema $^{1}$ \\ ${ }^{1}$ Laboratory of Transport Phenomena, Faculty of Mechanical and Process Engineering, USTHB, B.P. 32, El-Alia Bab- \\ Ezzouar, 16111 Algiers, Algeria
}

\begin{abstract}
Mixed convection heat and mass transfer in an inclined square cavity partially heated is numerically studied in the present paper. The cavity is filled with a non-Newtonian pseudoplastic fluid. The governing equations are solved numerically using the finite volume method. The velocitypressure coupling is achieved using the SIMPLER algorithm. This study focuses on the effect of some parameters, namely, the Richardson number and the power law index, on the flow pattern as well as on heat and mass transfer rates. The results indicate that the increase of the Richardson number decreases both heat and mass transfer rates. However, the latter are improved with the increase of the power law index whatever the value of the Richardson number.
\end{abstract}

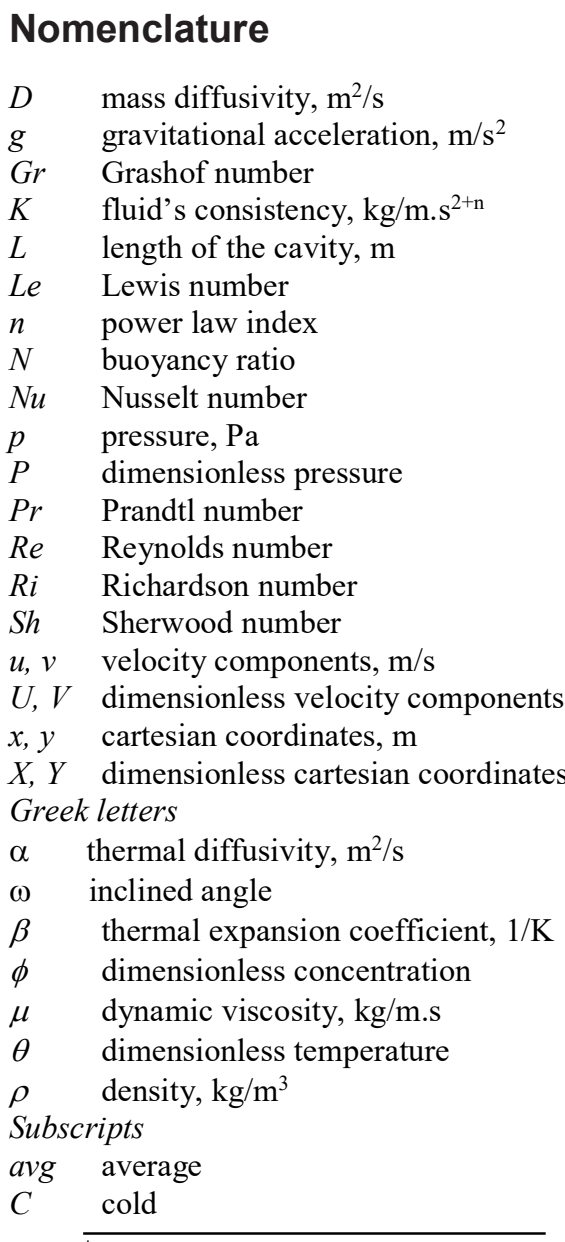

*Email: nihale.toudja@gmail.com

$\begin{array}{ll}H & \text { hot } \\ S & \text { solutal } \\ T & \text { thermal }\end{array}$

\section{Introduction}

Flow and heat transfer analysis in lid-driven cavities is one of the most widely studied problems in thermofluids areas. Numerous investigations have been conducted in the past on lid-driven cavity flow and heat transfer by considering various combinations of the imposed temperature gradients and cavity configurations [1]. In fact, the driven cavity configuration is encountered in many practical engineering and industrial applications such as materials processing, flow and heat transfer in solar ponds, reservoirs and cooling ponds, metal coating and so on. Moreover, the mentioned applications are usually combined with mass transfer. Kefayati [1] studied numerically, by means of the Lattice Boltzmann method, the double diffusion mixed convection of a non-Newtonian pseudoplastic fluid in a cavity. The results indicate that the increase in the Richardson number decreases heat and mass transfer. Nayak et al. [2] studied the thermosolutal mixed convection of a shear thinning fluid due to partially active mixed zones within a lid-driven cavity. Their results reveal that the location and the length of the heating and cooling zones have a significant contribution on the flow, heat and mass transfer. The rate of heat transfer is found to be maximum for a minimum heat source length and a maximum power law index. Kefayati [3] used the lattice Boltzmann method to study the 
double diffusive natural convection and entropy generation of power-law fluids in an inclined porous cavity with Soretand Dufour effects. The results indicate that the augmentation of the Darcy number enhances heat and mass transfer for different power-law indexes. The heat transfer is improved with the rise of the Dufour parameter and the mass transfer enhances as the Soret parameter increases for different power-law indexes and thermal Rayleigh numbers.

Chen et al. [4] studied numerically the double diffusive natural convection in a square cavity filled with a nanofluid. Their work tries to reveal the characteristics of the double diffusive natural convection over a wide range, from laminar to turbulent regimes, with the aid of numerical experiments. They observed that the behavior of the nanofluid in the laminar regime is different from that in the turbulent one.

In the present study, the double-diffusive mixed convection of a non-Newtonian pseudoplastic fluid in a partially heated square cavity inclined of an angle $\omega$, is numerically investigated. The study focuses on the effect of pertinent parameters, such as the Richardson number and the power law index, on the flow pattern as well as heat and mass transfer.

\section{Analysis and modelling}

\subsection{Physical and mathematical model}

The geometry of the present problem is shown in Figure 1. It consists of a two-dimensional cavity with the height L. The cavity is filled with a non-Newtonian pseudoplastic fluid. The bottom wall is inclined at an angle $\omega=120^{\circ}$ with respect to the horizontal. The portion of $\mathrm{L} / 3$ of the bottom of the left wall is maintained at high temperature and concentration $\left(\mathrm{T}_{\mathrm{H}}\right.$, $\mathrm{C}_{\mathrm{H}}$ ) while the $\mathrm{L} / 3$ of the top of the right wall is kept at low temperature and concentration $\left(\mathrm{T}_{\mathrm{C}}, \mathrm{C}_{\mathrm{C}}\right)$. The remaining portions of the left and the right walls as well as the two horizontal walls are adiabatic and impermeable. The horizontal top wall is driven from the left to the right by a constant velocity $\mathrm{U}_{0}$.

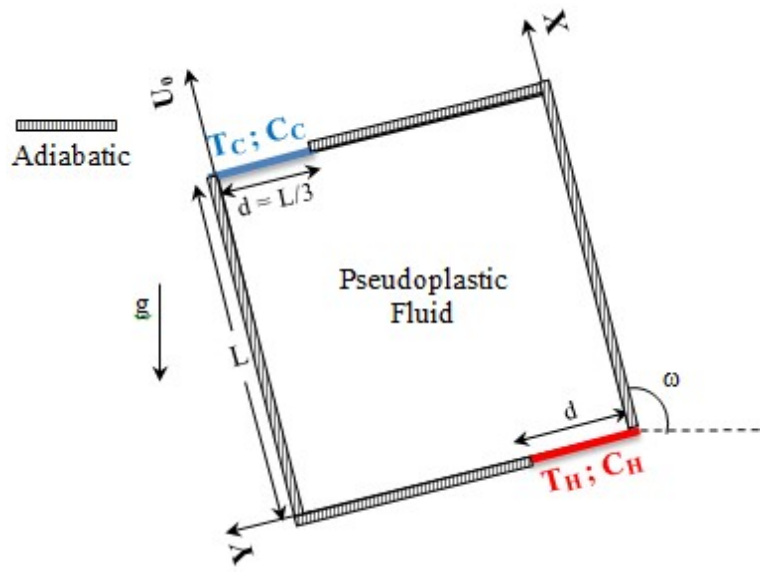

Fig. 1. Geometry of the present study.

\subsection{Governing equations}

The governing equations are: Continuity, $\mathrm{X}$ and $\mathrm{Y}$ momentum, energy and diffusion. They are given, respectively in dimensionless form as follows:

$$
\frac{\partial U}{\partial X}+\frac{\partial V}{\partial Y}=0
$$

$$
\begin{aligned}
& U \frac{\partial U}{\partial X}+V \frac{\partial U}{\partial Y}=-\frac{\partial P}{\partial X}+\frac{1}{\operatorname{Re}} \\
& \times {\left[2 \frac{\partial}{\partial X}\left(\frac{\mu_{a}}{K} \frac{\partial U}{\partial X}\right)+\frac{\partial}{\partial Y}\left(\frac{\mu_{a}}{K}\left(\frac{\partial U}{\partial Y}+\frac{\partial V}{\partial X}\right)\right)\right] } \\
&+R i(\theta+N \phi) \sin \omega \\
& U \frac{\partial V}{\partial X}+V \frac{\partial V}{\partial Y}=-\frac{\partial P}{\partial Y}+\frac{1}{\operatorname{Re}} \\
& \times\left[2 \frac{\partial}{\partial Y}\left(\frac{\mu_{a}}{K} \frac{\partial V}{\partial Y}\right)+\frac{\partial}{\partial X}\left(\frac{\mu_{a}}{K}\left(\frac{\partial U}{\partial Y}+\frac{\partial V}{\partial X}\right)\right)\right] \\
&+R i(\theta+N \phi) \cos \omega \\
& U \frac{\partial \theta}{\partial X}+V \frac{\partial \theta}{\partial Y}=\frac{1}{\operatorname{Re} \operatorname{Pr}}\left(\frac{\partial^{2} \theta}{\partial X^{2}}+\frac{\partial^{2} \theta}{\partial Y^{2}}\right) \\
& U \frac{\partial \phi}{\partial X}+V \frac{\partial \phi}{\partial Y}=\frac{1}{L e \operatorname{RePr}}\left(\frac{\partial^{2} \phi}{\partial X^{2}}+\frac{\partial^{2} \phi}{\partial Y^{2}}\right)
\end{aligned}
$$

For a purely-viscous non-Newtonian fluid which follows the Ostwald-De Waele (i.e. power-law) model, the apparent viscosity $\mu_{\mathrm{a}}$ is written in dimensionless form as follows:

$$
\mu_{a}=K\left\{2\left[\left(\frac{\partial U}{\partial X}\right)^{2}+\left(\frac{\partial V}{\partial Y}\right)^{2}\right]+\left(\frac{\partial V}{\partial X}+\frac{\partial U}{\partial Y}\right)^{2}\right\}^{(n-1) / 2}
$$

The non-dimensionalized governing equations (1-5) were obtained using the following dimensionless quantities:

$$
\begin{gathered}
X=\frac{x}{L}, Y=\frac{y}{L}, U=\frac{u}{U_{0}}, V=\frac{v}{U_{0}}, P=\frac{p}{\rho U_{0}^{2}}, \\
\theta=\frac{T-T_{C}}{T_{H}-T_{C}}, \phi=\frac{C-C_{C}}{C_{H}-C_{C}}
\end{gathered}
$$

The dimensional analysis generates the following dimensionless numbers:

$$
\begin{gathered}
\operatorname{Re}=\frac{\rho U_{0} L}{\mu_{a}}, R i=\frac{g \beta \Delta T L}{U_{0}^{2}}=\frac{G r_{T}}{\operatorname{Re}^{2}}, \operatorname{Pr}=\frac{\mu_{a}}{\rho \alpha}, L e=\frac{\alpha}{D}, \\
N=\frac{G r_{s}}{G r_{T}}
\end{gathered}
$$

The dimensionless boundary conditions of the problem are:

$$
\begin{gathered}
Y=0, \quad 0 \leq X \leq 1, \quad U=V=0, \quad \frac{\partial \theta}{\partial Y}=\frac{\partial \phi}{\partial Y}=0 \\
Y=1, \quad 0 \leq X \leq 1, \quad U=1, V=0, \quad \frac{\partial \theta}{\partial Y}=\frac{\partial \phi}{\partial Y}=0 \\
X=0, \quad U=V=0, \quad \frac{\partial \theta}{\partial X}=\frac{\partial \phi}{\partial X}=0,(\text { except the hot } \\
\text { portion) }
\end{gathered}
$$




$$
\begin{aligned}
& X=0, \quad 0 \leq Y \leq 1 / 3, \quad \theta=\phi=1 \quad \text { (Hot portion) } \\
& X=1, \quad U=V=0, \quad \frac{\partial \theta}{\partial X}=\frac{\partial \phi}{\partial X}=0,(\text { except the cold } \\
& \text { portion) } \\
& X=1, \quad 2 / 3 \leq Y \leq 1, \quad \theta=\phi=0 \text { (Cold portion) }
\end{aligned}
$$

The local and the average Nusselt numbers at the hot wall are calculated as:

$$
\begin{aligned}
& N u_{h}=\left(-\frac{\partial \theta}{\partial X}\right)_{X=0} \\
& N u_{\text {avg }}=\int_{0}^{1} N u_{h} d Y
\end{aligned}
$$

The local and the average Sherwood numbers at the hot (and concentrated) wall are calculated as:

$$
\begin{gathered}
S h_{h}=\left(-\frac{\partial \phi}{\partial X}\right)_{X=0} \\
S h_{\text {avg }}=\int_{0}^{1} S h_{h} d Y
\end{gathered}
$$

\subsection{Numerical modeling and validation of the computer code}

The finite volume formulation, given by Patankar [5] on a staggered grid, is applied for the discretization of both governing equations and boundary conditions. The coupling between pressure and velocity is achieved using the SIMPLER algorithm.

The validation of our computer code is performed by comparing our results with those obtained by Kefayati [1] who investigated the double-diffusive mixed convection of pseudoplastic fluids in a two sided liddriven cavity. The comparison of the structure of the streamlines, isotherms and isoconcentrations (Figure 2) and the distribution of the local Nusselt number along the hot wall for different values of the power law index (Figure 3) show good agreement between the results. This allows us to validate our computer code.

\section{Streamlines Isotherms Isoconcentrat- ions}

(a)
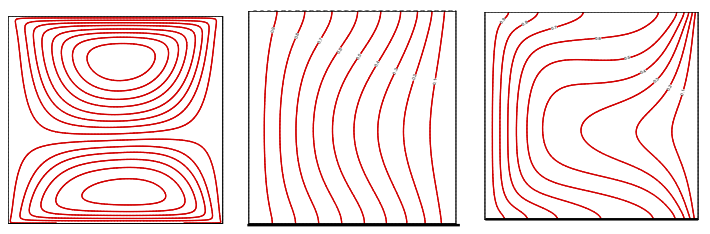

(b)
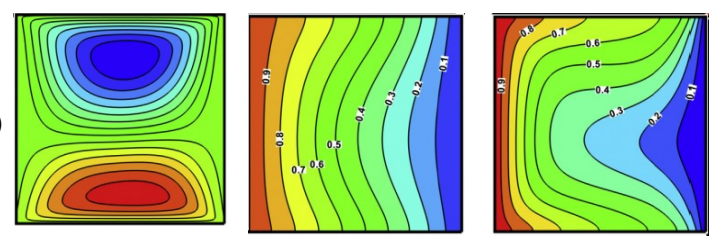

Fig. 2. Comparison of the streamlines, the isotherms and isoconcentrations between (a) the present results and (b) numerical results by Kefayati. [2] at $\mathrm{Ri}=1, \mathrm{~N}=1, \mathrm{Le}=5$ and $\mathrm{n}=0.6$.

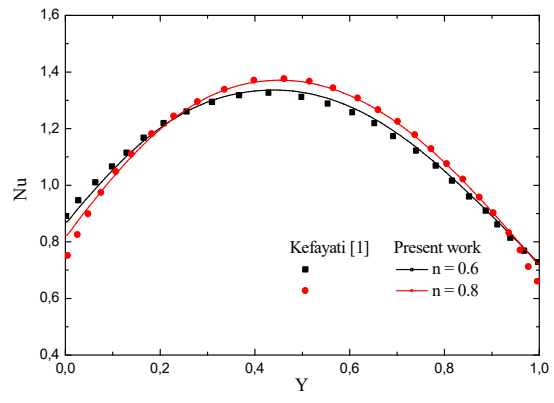

Fig. 3. Local Nusselt number on the hot wall for different values of the power law index at $\mathrm{Ri}=1, \mathrm{~N}=1$ and $\mathrm{Le}=5$.

\section{Results and discussion}

Figure 4 shows the streamlines, isotherms, and isoconcentrations for different values of the power law index and the Richardson number and for $\operatorname{Le}=5, \operatorname{Pr}=1$, $\mathrm{N}=1, \omega=120^{\circ}$.

For $\mathrm{Ri}=0.01$, i.e. the dominant forced convection mode, the streamlines are represented by eccentric vortexes located in the region adjacent to the lid-driven wall because of the position of active portions, and are oriented towards the translation velocity direction of the latter. The central vortexes move to the center as the power law index increases. However, for the case of a mixed convection, $\mathrm{Ri}=1$, since the natural convection effects appear, the streamlines are still located near the lid-driven wall but are concentric and get away from the lid-driven wall as the power law index increases. They expand gradually with the increase of the power law index which confirms the enhancement of the natural convection.

Whatever the convection mode and the value of the power law index, because of the angle of inclination of the enclosure, the isotherms and the isoconcentrations show that the hot and concentrated fluid migrates upward from the vicinity of the hot portion and is cooled down gradually as approaching the cold portion. At the contact of the latter, the cold and poor fluid falls down.

For $\mathrm{Ri}=0.01$, the rise of the power law index leads to the decrease of both thermal and solutal boundary layers thickness which increases both temperature and concentration gradients near the active portions of the vertical walls. Indeed, for $n=0.2$, the behavior of the isotherms and the isoconcentrations corresponds to a conduction and a diffusion, respectively, where both the isotherms are nearly parallel to the active walls. However, the thermal convection process increases slightly due to the rise of the power law index. This pattern is also observed in the isoconcentrations where the convection of mass transfer enhances significantly because of the increase of the power law index. For $\mathrm{Ri}=$ 1 , the effect of the forced convection decreases substantially and the increase of the power law index does not alter the isotherms and isoconcentrations considerably. 

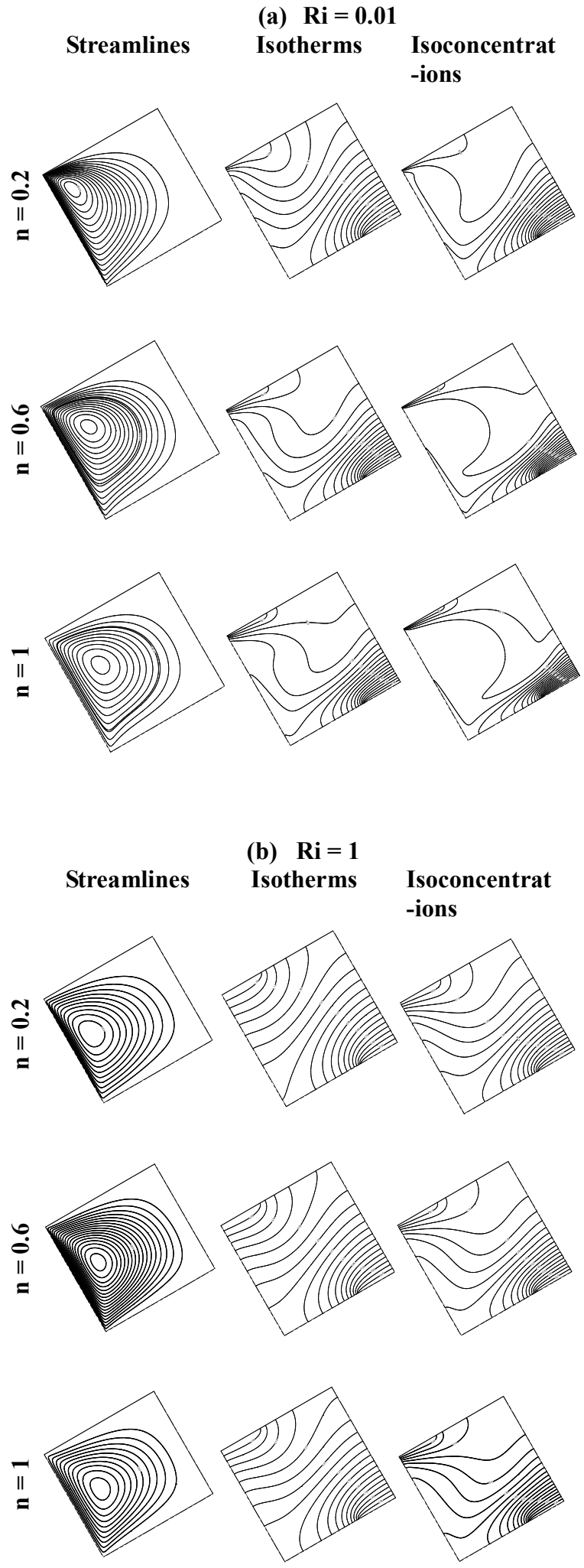

Fig. 4. Streamlines, isotherms, and isoconcentrations for different values of the power law index and Richardson number. $\mathrm{Le}=5, \operatorname{Pr}=1, \mathrm{~N}=1$ and $\omega=120^{\circ}$. (a) $\mathrm{Ri}=0.01$, (b) $\mathrm{Ri}=1$.
Figure 5 displays the vertical velocity component and the temperature profiles in the midline of the cavity $(\mathrm{Y}=$ 0.5 ) for different values of the power law index and for the cases of dominant forced and mixed convection. It is obvious that the velocity in the midline of the cavity increases as the power law index increases. In fact, it demonstrates that the fluid moves easily and it results, eventually, in convection process enhancement. Furthermore, it is observed that the variation in the power law index extremely affects the dimensionless temperature profiles. Indeed, as the power law index decreases the temperature profiles become flattened since for very low values of this rheological parameter, a conduction heat transfer mode is observed.

A different behavior is observed for the mixed convection mode $(\mathrm{Ri}=1)$. Indeed, the vertical velocity profiles show a perfect symmetry in comparison to the case of a dominant forced convection since in this case, the buoyancy and the shear forces are of equal intensity. Regarding the dimensionless temperature profiles, the power law index variation has no significant effect for this case.
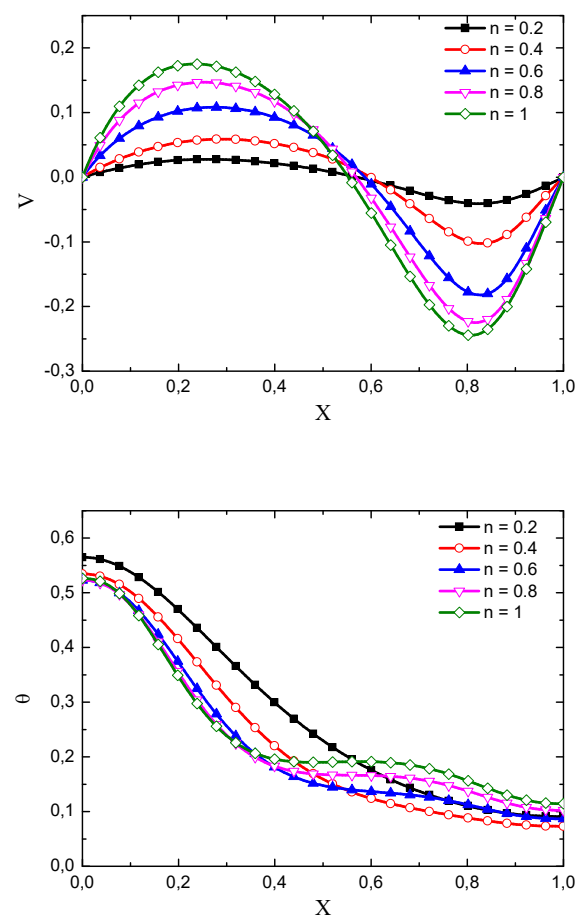

(a) $\mathrm{Ri}=\mathbf{0 . 0 1}$ 

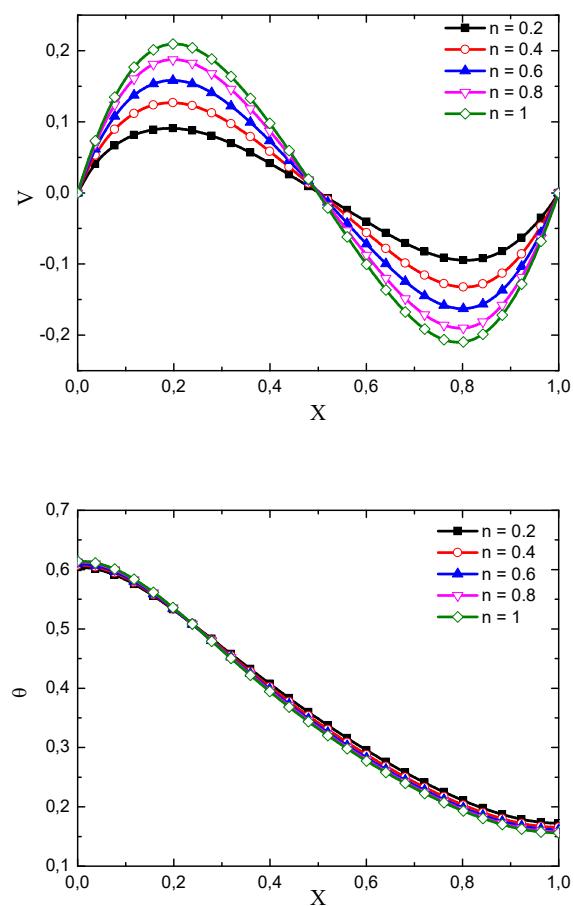

(b) $\mathrm{Ri}=1$

Fig. 5. Vertical velocity and temperature profiles at $\mathrm{Y}=0.50$ for different values of the power law index. $\mathrm{Le}=5, \operatorname{Pr}=1, \mathrm{~N}=1$ and $\omega=120^{\circ}$. (a) $\mathrm{Ri}=0.01$, (b) $\mathrm{Ri}=1$

Figure 6 (a)-(b) shows the average Nusselt and Sherwood numbers on the hot wall according to the power law index and for different values of the Richardson number. It can be seen that the increase of the power law index and the decrease of the Richardson number enhance both heat and mass transfer within the enclosure. Furthermore, the effect of the increase of the power law index on the average Nusselt and Sherwood numbers is more visible for the case of a dominant forced convection compared to the case of a mixed convection where the variation is not significant.

(a)

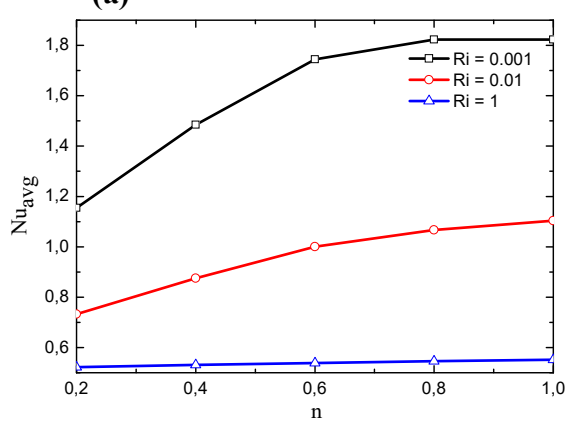

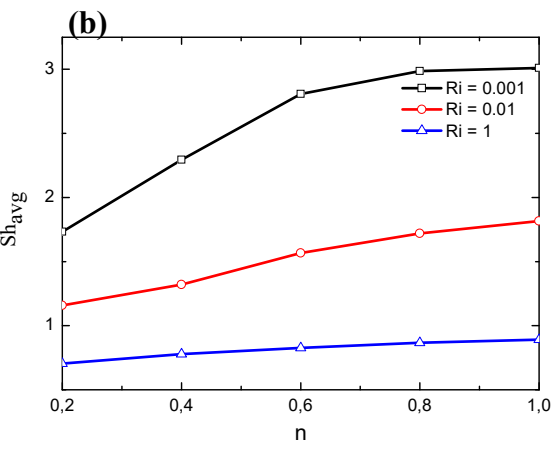

Fig. 6. Average (a) Nusselt and (b) Sherwood numbers according to the power law index and for different values of the Richardson number. $\mathrm{Le}=5, \operatorname{Pr}=1, \mathrm{~N}=1$ and $\omega=120^{\circ}$.

\section{Conclusions}

A double-diffusive mixed convection of pseudoplastic fluids within an inclined square cavity partially heated, is investigated numerically in this paper. The equations governing the flow, the heat and mass transfer are solved numerically using the finite volume method. The numerical results are obtained for different values of the Richardson number and the power law index.

From the above discussion, the main conclusions can be summarized as follows:

(a) The velocity in the middle of the cavity increases with increasing the power law index.

(b) Heat and mass transfer declines with the increase of the Richardson number for different values of the power law index.

(c) Heat and mass transfer are enhanced as the power law index increases whatever the heat convection mode. However, the dominant forced convection case exhibits the more significant improvement.

At the end of this study, some perspectives could be considered such as varying the angle of the inclined cavity in order to choose an optimal configuration providing the best heat and mass exchanges.

\section{References}

1. GH.R. Kefayati, J Taiwan Inst Chem Eng, 45, 21222139 (2014)

2. A.K. Nayak, A. Haque, A Banerjee, Int J Heat Mass Transfer, 106, 686-707 (2017)

3. GH.R. Kefayati, Int J Heat Mass Transfer, 94, 539581 (2016)

4. S. Chen, B. Yang, K H. Luo, X. Xiong, C. Zheng, Int J Heat Mass Transfer, 95, 1070-1083 (2016)

5. S.V. Patankar, Numerical Heat Transfer and Fluid Flow, McGraw-Hill, New York, (1980) 